\title{
Frequency-Dependent Impedance Responses of ZnO Using UV Light
}

\author{
Jiaqi Cheng ${ }^{1}$ and Kristin M. Poduska $\oplus^{1,2, *, z}$ \\ ${ }^{I}$ Department of Chemistry, Memorial University of Newfoundland, St. John's, NL A1B 3X7, Canada \\ ${ }^{2}$ Department of Physics and Physical Oceanography, Memorial University of Newfoundland, St. John's, NL A1B 3X7, \\ Canada
}

\begin{abstract}
Impedance spectroscopy data show that polycrystalline $\mathrm{ZnO}$ films can show either increases or decreases in their effective resistances after UV exposure, depending on the frequency of the applied AC excitation. Simple equivalent circuit models, based on resistance $(R)$ and capacitance $(C)$ in parallel, are sufficient to confirm the observed experimental trends. Simulated data demonstrate that that arbitrary $R$ and $C$ values will not produce the sign change, but that typical resistance and capacitance characteristics for photoconductive semiconductors like $\mathrm{ZnO}$ can cause the sign change. These results suggest that it could be desirable to manipulate the $R$ and $C$ values of photodetector materials to either control - or eliminate - such frequency-dependent $\mathrm{UV}$ responses.

(C) The Author(s) 2019. Published by ECS. This is an open access article distributed under the terms of the Creative Commons Attribution Non-Commercial No Derivatives 4.0 License (CC BY-NC-ND, http://creativecommons.org/licenses/by-nc-nd/4.0/), which permits non-commercial reuse, distribution, and reproduction in any medium, provided the original work is not changed in any way and is properly cited. For permission for commercial reuse, please email: oa @electrochem.org. [DOI: 10.1149/2.0041901jss]
\end{abstract}

(cc) BY-NC-ND

Manuscript submitted August 27, 2018; revised manuscript received December 31, 2018. Published January 10, 2019.

The conductivity changes that occur in some materials upon exposure to UV light has sparked a wide range of applications in optoelectronics, ${ }^{1}$ flame sensing, ${ }^{2}$ and biosensing. ${ }^{3}$ Among the many wide-bandgap semiconductors that have been explored for UV sensors, ${ }^{2} \mathrm{ZnO}$ has attracted extensive attention because of its relatively low cost, ease of production, and rich surface chemistry. ${ }^{1,3}$

The mechanism behind electrical resistance changes upon exposure to light can vary among different materials. ${ }^{2}$ For $\mathrm{ZnO}$, studies have shown that surface states play a dominant role in photoconductivity. ${ }^{4,5}$ When light shines on $\mathrm{ZnO}$, it absorbs photons that generate electron-hole pairs in the bulk. These photo-generated holes migrate to the surface and neutralize adsorbed oxygen ions $\left(\mathrm{O}_{2}^{-}\right)$that form readily forms $\mathrm{ZnO}$ surfaces under ambient conditions. This causes $\mathrm{O}_{2}$ desorption; the photo-generated electron that remains in the $\mathrm{ZnO}$ causes a conductivity increase. Thus, based on this mechanism, the photoresponse of $\mathrm{ZnO}$ is controlled by the $\mathrm{O}_{2}$ adsorption/desorption equilibrium at surfaces. This has led to a flourish of research activity related to $\mathrm{ZnO}$ powders, wires, and rods because their large surface-volume ratios tend to cause a stronger photoconductive response..$^{2,6,7}$

Photoconductivity of polycrystalline $\mathrm{ZnO}$ films and nanostructures have been explored not only with direct current (DC) excitations, but also as a function of alternating current (AC) excitations using impedance spectroscopy. AC-dependent conductivity trends are particularly important for assessing how the $\mathrm{ZnO}$ would function in a capacitance-based sensor, since the time required for capacitor charging and discharging could influence sensor response times. Impedance spectra of nanocrystalline $\mathrm{ZnO}$ films taken before and during $\mathrm{UV}$ exposure, showed that small average grain sizes, high porosity, and specific kinds of surface chemistries increase DC and AC conductivity. ${ }^{8}$ More recently, electrochemical impedance spectroscopy was paired with gas chromatography mass spectrometry (GC-MS) to probe the chemical origin of the UV responses of $\mathrm{ZnO}$ nano-crystalline film in an ambient environment. ${ }^{5}$ That work showed that $\mathrm{ZnO}$ impedance changes are correlated with desorption of $\mathrm{O}_{2}$, water and other organic byproducts from $\mathrm{ZnO}$ surfaces via $\mathrm{UV}$ induced photochemistry.

In this study, we show an interesting and surprising effect: depending on the AC excitation frequency, UV light can cause either an increase or a decrease in its apparent resistance. We demonstrate that this behavior is consistent with UV-induced changes in film's resistance, based on simulated data based on equivalent circuit modeling. Based on the simplicity of the origin effect, we proposed that it could be generalizable to other photoconductive materials.

\footnotetext{
*Electrochemical Society Member.
}

zE-mail: kris@mun.ca

\section{Experimental}

$\mathrm{ZnO}$ film preparation.- $\mathrm{ZnO}$ powder was synthesized using a room-temperature solid-state metathesis reaction that is described in an earlier publication. ${ }^{9}$ The starting materials, $\mathrm{ZnCl}_{2}$ and $\mathrm{NaOH}$, were ground into fine powders using an agate mortar and pestle. They were then mixed together (1:2 molar ratio) in a glass beaker, during which an exothermic reaction produced a white paste. Filtering with ultrapure water (Barnstead Nanopure, 18.2 M $\Omega \cdot \mathrm{cm}$ ) removed the $\mathrm{NaCl}$ by-product, leaving the desired $\mathrm{ZnO}$ solid. After air drying for several hours, the powder was heated for 8 hours at $500^{\circ} \mathrm{C}$, ramping and cooling at a rate of $100^{\circ} \mathrm{C}$ per hour. A $\mathrm{ZnO}$ slurry was then prepared from $0.10 \mathrm{~g}$ of the annealed powder that was dispersed in $2 \mathrm{~mL}$ of $1: 1$ ethanol/water mixture. After $30 \mathrm{~s}$ of ultrasonication, the slurry was spread by hand over an electrically conductive indium tin oxide (ITO) coated glass slide (8-12 $\Omega$, Delta Technology). A second ITO electrode was pressed on top, held in place by binder clips, to sandwich the $\mathrm{ZnO}$ film.

Photoresponse measurements.-Prior to the measurements, $\mathrm{ZnO}$ films were allowed to equilibrate for $24 \mathrm{~h}$ in a dark box under steady humidity levels that were controlled using saturated salt solutions. ${ }^{10,11}$ All measurements were conducted at room temperature $\left(22 \pm 2^{\circ} \mathrm{C}\right)$. The UV lamp (365 nm emission, Model UVGL-25, UVP Inc.) had an intensity of $1.6 \mathrm{~mW} \mathrm{~cm}^{-2}$ at a distance of $5 \mathrm{~cm}$.

Impedance spectroscopy was performed using a Princeton Applied Research potentiostat/galvanostat (Model 273A with Signal Recovery Model 5210 Lock-in Amplifier, input impedance 10 G $\Omega$, Power SUITE software). A sinusoidal AC potential (10 mV RMS amplitude, $0 \mathrm{~V}$ DC bias) was swept from $10^{5} \mathrm{~Hz}$ to $10^{-1} \mathrm{~Hz}$. DC film resistances were measured separately with an inductance-capacitance-resistance (LCR) meter (National Instruments) using a DC test current $(0.5 \mu \mathrm{A}$; meter range $10 \mathrm{M} \Omega$, resolution $10 \Omega$ ).

Equivalent circuit fitting of the EIS data was done manually by superimposing simulated spectra (EIS Spectrum Analyser freeware ${ }^{12}$ ) onto the raw data and iteratively adjusting parameters. All EIS data described in this work could be modeled well with a circuit containing only one resistor and one capacitor in parallel.

\section{Results and Discussion}

Electrical characterization.-Figure 1 shows representative examples of DC resistance changes when $\mathrm{ZnO}$ films are exposed to intermittent periods of UV exposure. UV illumination triggers an abrupt resistance decrease. Once the light is removed, the sample resistance returns to the original value after hundreds of seconds. Based on data 


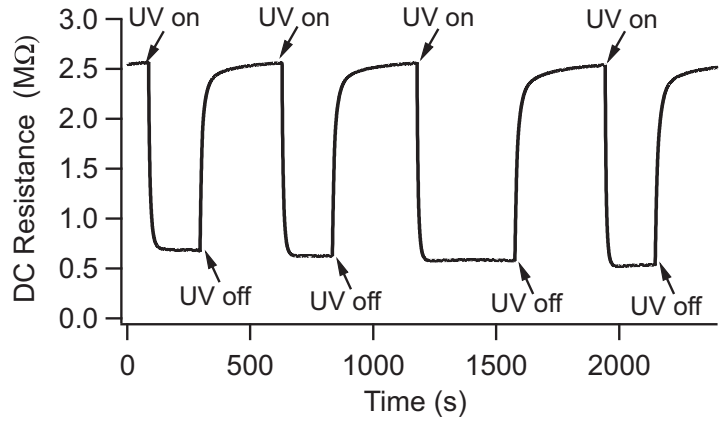

Figure 1. Representative resistance changes for a $\mathrm{ZnO}$ film in response to intermittent UV light exposure.

from ten comparably prepared samples, $\mathrm{ZnO}$ film resistances were $\mathrm{M} \Omega$-range values under dark conditions, and UV exposure reduced the resistance by factors of 2-10.

Fig. 2 compares representative Nyquist and Bode plots for the dark and light impedance behaviors of the $\mathrm{ZnO}$ films. Consistent with the DC resistance data, $Z_{r e}$ decreases after UV exposure in the low-frequency regime. $Z_{i m}$ has a more complicated response as a function of frequency, showing a maximum (labeled $f_{\max }$ in Figures $2 \mathrm{c}, 2 \mathrm{f})$. Above this frequency, $Z_{r e}$ is no longer frequency independent (labeled $f_{\max }$ in Figures $2 \mathrm{~b}, 2 \mathrm{e}$ ).
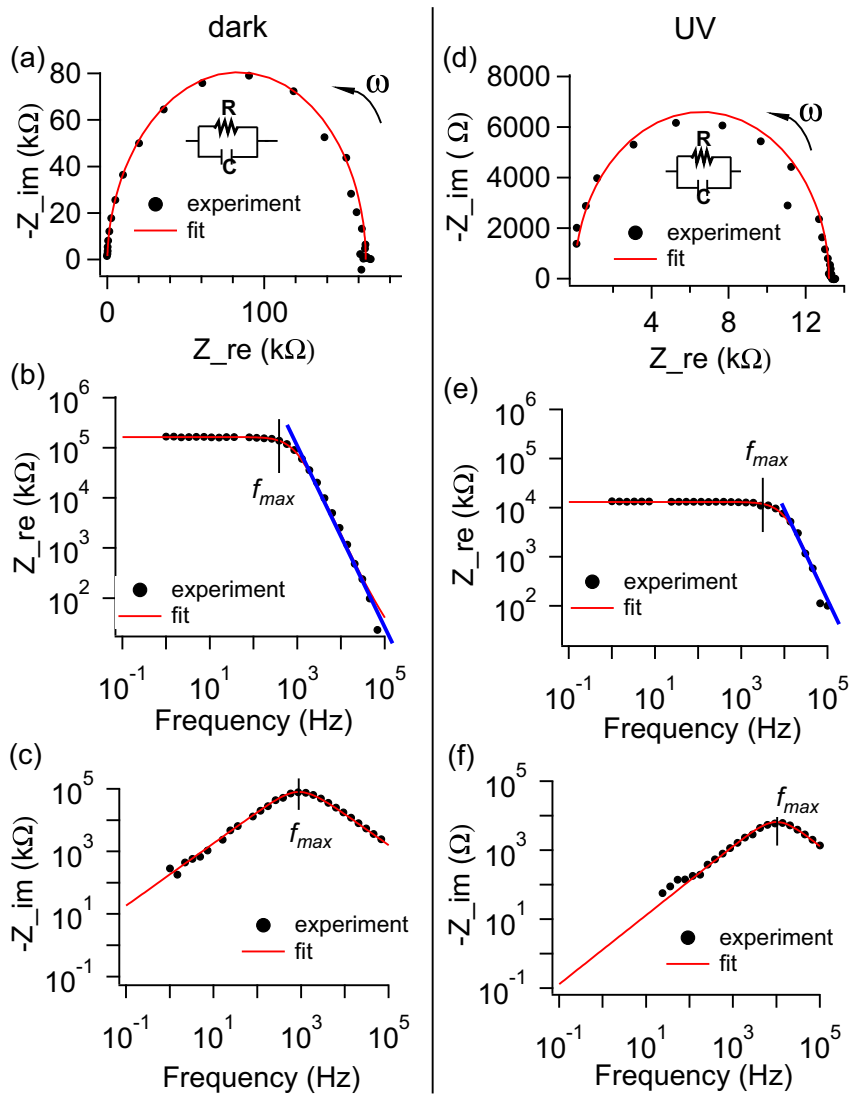

Figure 2. Representative EIS data in the dark (a-c) and under UV illumination (d-f). Experimental data are shown as black dots, and they were fit to an equivalent circuit that is shown in the insets of panels (a) and (d). Arrows in the Nyquist plots $(\mathrm{a}, \mathrm{d})$ indicate the direction of increasing frequency (ranging from $0.1 \mathrm{~Hz}$ to $100 \mathrm{kHz}$ ). Blue fit lines (b,e) highlight the linear portions of the $\log$ - $\log$ Bode plots for the high frequency range of $Z_{r e} \cdot f_{\text {max }}$ denotes the maximum frequency before which $Z_{r e}$ begins to decrease.
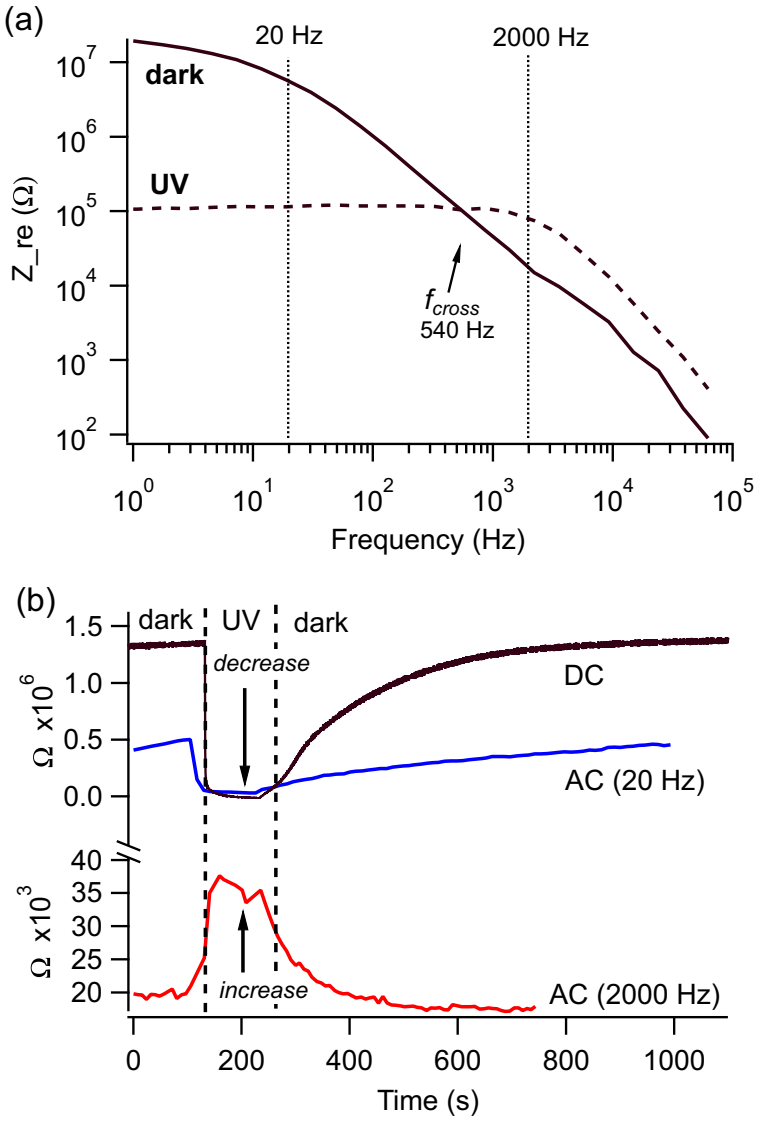

Figure 3. (a) Representative $Z_{r e}$ vs. $f$ data taken in the dark and under UV light, with their intersection point $\left(f_{\text {cross }}=540 \pm 50 \mathrm{~Hz}\right)$ indicated by an arrow. (b) Representative photoresponses under DC conditions (black), $f$ lower than $f_{\text {cross }}\left(20 \mathrm{~Hz}\right.$, shown in blue), and $f$ greater than $f_{\text {cross }}(2000 \mathrm{~Hz}$, shown in red). The arrows indicate that UV exposure causes an increase in $Z_{r e}$ for $2000 \mathrm{~Hz}$ excitation, but a decrease for DC or $20 \mathrm{~Hz}$ excitation.

An interesting phenomenon appears when one compares the $Z_{r e}$ vs. $f$ plots for dark and UV illumination conditions. Figure 3 a shows that, when these two Bode plots are overlaid, the spectra intersect at a frequency that we denote as $f_{\text {cross }}$. This crossing suggests that opposite signs for UV-induced resistance changes could occur, depending on the value of the test frequency. Data in Figure $3 \mathrm{~b}$ confirm this sign change. At frequencies smaller than $f_{\text {cross }}$ (either DC or $20 \mathrm{~Hz} \mathrm{AC}$ ), the magnitude of $Z_{r e}$ is larger in the dark than in UV. However, for frequencies above $f_{\text {cross }}(2000 \mathrm{~Hz}), Z_{r e}$ is larger during UV exposure. Thus, UV exposure can cause either an increase or a decrease in the film resistance, depending on the relation between the excitation frequency and $f_{\text {cross }}$.

Modelling frequency-dependent photoresponses.-We used equivalent circuit modeling to show that $f_{\text {cross }}$, and the associated sign change in the resistive photoresponse, is a robust phenomenon.

Simulated Bode spectra were based on a parallel $R C$ circuit (Figure 4 inset), with varying $R$ and $C$ values $(R=10-1000 \mathrm{k} \Omega$ and $C=$ $0.1-100 \mathrm{nF}$ ) that included the range we measured for our $\mathrm{ZnO}$ films $(R=10-1000 \mathrm{k} \Omega$ and $C=0.4-1.4 \mathrm{nF})$.

The simulations clearly show that changing $R$ and $C$ values result in a shift of $Z_{r e}$ spectra. Fig. 4a presents simulation results after decreasing $C$ while keeping $R$ constant. This manipulation leads to a shift of $f_{\max }$ toward higher frequencies and does not cause $f_{\text {cross }}$. Fig. 4b shows another scenario: decreasing $R$ while increasing $C$ proportionally, in order to keep the ratio $R C$ constant. In this case, $Z_{r e}$ drops over the whole frequency range without intersecting the original spectrum. Fig. $4 \mathrm{c}$ shows that $f_{\text {cross }}$ occurs when $R$ decreases. 
(a)

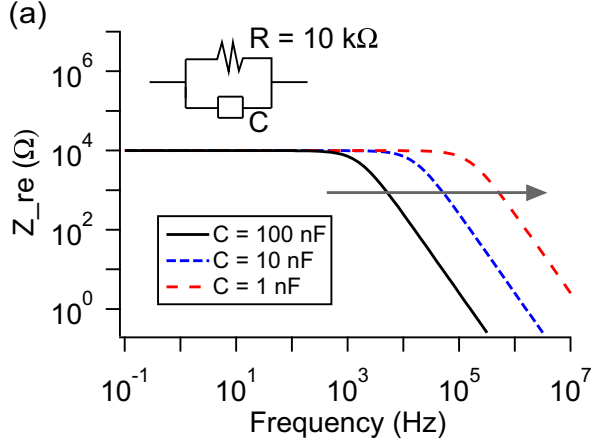

(b)

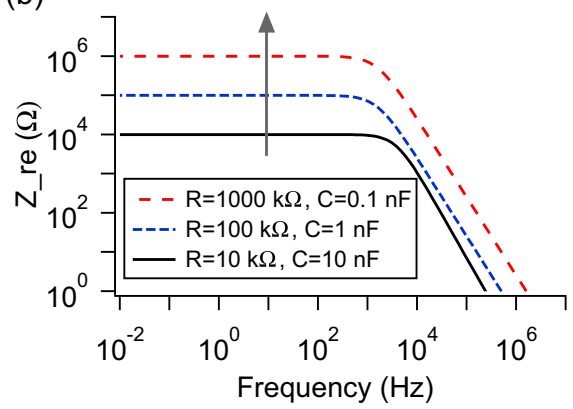

(c)

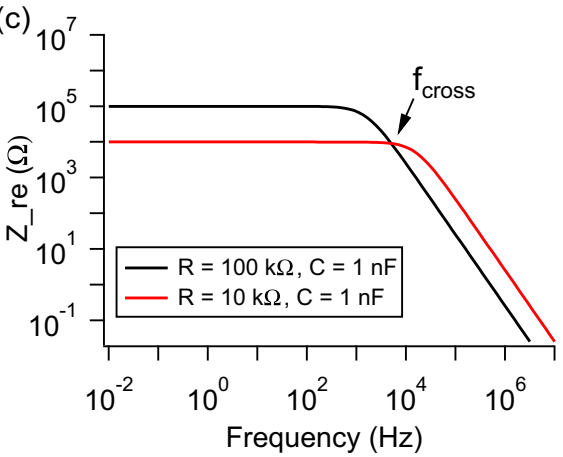

Figure 4. Simulated $Z_{r e}$ vs. $f$ data based on a parallel $R C$ circuit shown in (a). In (a), $C$ decreased while $R$ was constant. In (b), $R$ decreased while keeping the ratio $R C$ constant. In (c), $f_{\text {cross }}$ occurs when $R$ decreases while $C$ is constant.

Based on these simulations, the occurrence of $f_{\text {cross }}$ for a photoresponsive film (based on parallel $R C$ equivalent circuit behavior) must satisfy two conditions. First, $R_{\text {dark }}>R_{U V}$. Second, $R_{\text {dark }} \times C_{\text {dark }}$ $>R_{U V} \times C_{U V}$. These conditions would be easy to satisfy in many $\mathrm{ZnO}$ films. A DC resistance decrease during UV exposure is consistent with the $\mathrm{ZnO}$ photoresponse mechanism proposed by others. ${ }^{4,5,13}$ This means that the first criterion will typically be met in $\mathrm{ZnO}$ films. The second criterion would then also be satisfied if UV exposure did not cause a significant capacitance increase.

Relating these simulations to experimental findings, equivalent circuit fits of EIS data show that our ZnO films satisfy the two conditions necessary for $f_{\text {cross }}$ to occur. Figure 2 shows that both dark and UV impedance data can be fit well with a simple parallel $R C$ equivalent circuit. Based on $R$ and $C$ values extracted from these fits, $\mathrm{UV}$ exposure affects the resistance of the $\mathrm{ZnO}$ films much more than the capacitance. For example, the data shown in Figure 2 indicate a resistance change from $150 \pm 5 \mathrm{k} \Omega$ dark compared to $13.2 \pm 0.2 \mathrm{k} \Omega$ for UV, while the capacitance shows no appreciable increase $(1.0 \pm$ $0.2 \mathrm{nF}$ for dark compared with $1.2 \pm 0.2 \mathrm{nF}$ for $\mathrm{UV}$ ). The fits yielding these $R$ and $C$ values are superimposed over the experimental data in Figure 2.

Equivalent circuit modeling also helps to explain the origin of $f_{\max }$ in Figures 2c, 2f. For a parallel $R C$ circuit, the behavior of $Z_{i m}$ as a function of frequency follows the relation:

$$
Z_{i m}(f)=-R \frac{2 \pi f \tau}{1+(2 \pi f \tau)^{2}}
$$

where $\tau$ is the capacitive time constant. This is related to $f_{\max }$ by:

$$
\tau=R C=\frac{1}{2 \pi f_{\max }}
$$

In log-log form, Eq. 1 can be re-written as:

$$
\log \left[Z_{i m}\right]=\log [f]+\log [-2 \pi \tau R]-\log \left[1+(2 \pi f \tau)^{2}\right]
$$

Written in this form, it is evident that the maximum value of $Z_{i m}$ occurs when the applied frequency $f$ matches the inverse of the time constant $\tau$; this is $f_{\text {max }}$. Thus, a peak in the $-Z_{\text {im }}$ Bode plot will occur for any system that can be modeled with a parallel $R C$ equivalent circuit.

In a similar way, a parallel $R C$ circuit explains why the real component of the impedance $Z_{r e}$ also changes behavior at $f_{\max }$. For an $R C$ equivalent circuit,

$$
Z_{r e}(f)=\frac{R}{1+(2 \pi f \tau)^{2}}
$$

In log-log form:

$$
\log \left[Z_{r e}\right]=\log [R]-\log \left[1+(2 \pi f \tau)^{2}\right]
$$

For low frequencies $\left(f \ll f_{\max }\right)$, the second term in Equation 5 is negligible, meaning that $Z_{r e}$ is related only to the resistance $R$ and is independent of $f$. However, at high frequencies $\left(f \gg f_{\max }\right)$, $Z_{r e} \rightarrow R /(2 \pi f)^{2}$. When plotted on a log-log scale (Figs. 2b, 2e), the manifestation of $f_{\max }$ is the frequency at which the roll-off in $Z_{r e}$ begins.

Given that the appearance of $f_{\text {cross }}$ is a relatively robust phenomenon for photoresponsive films, it is important to note some practical considerations. Although $f_{\text {cross }}$ is very consistent for a given $\mathrm{ZnO}$ film (typically within $10 \%$ ), experimentally derived values of $f_{\text {cross }}$ for prepared under similar conditions can vary from $500 \mathrm{~Hz}-10 \mathrm{kHz}$. Preliminary experiments using other methods of producing $\mathrm{ZnO}$ (such as molten salt or solvothermal syntheses ${ }^{14}$ ) also yields $f_{\text {cross }}$ values within this range, but the situation can be more complicated if the impedance response is not accurately described by a single parallel $R C$ circuit. These variances from film to film are not surprising given that resistance is affected by variations in film thickness, as well as environmental factors such as humidity. Furthermore, $\mathrm{ZnO}$ resistivity can vary from $10^{-4}$ to $10^{8} \Omega \cdot \mathrm{cm}$, even in nominally undoped films, due to changes in native defects. ${ }^{15}$ For these reasons, the optimal AC operating frequency for each photoresponsive film would need to be calibrated based on its own measured $f_{\text {cross }}$ value.

\section{Conclusions}

We report an interesting effect in the frequency-dependent photoresponses of $\mathrm{ZnO}$ films: the sign reverses between low and high excitation frequencies. Our impedance data and modeling indicates that this frequency-dependent UV response can be caused by a resistance change that is induced by UV exposure, as long as there is no appreciable increase in film capacitance. Furthermore, impedance spectroscopy can be used to identify the threshold between low and high frequencies $\left(f_{\text {cross }}\right)$ at which the change in the sign of the UV response occurs. Our findings suggest that it could be desirable to manipulate the $R$ and $C$ values of photodetector materials to either control - or eliminate - these frequency-dependent UV responses, which would be particularly relevant for capacitance-based sensors.

\section{Acknowledgment}

Tanzir Ahmed and Erica Hayward assisted in validating sample preparation and measurement protocols. KMP acknowledges NSERC (Canada) Discovery Grant program for funding. 


\section{ORCID}

Kristin M. Poduska (D https://orcid.org/0000-0003-4495-0668

\section{References}

1. S.-J. Young, C.-C. Yang, and L.-T. Lai, J. Electrochem. Soc., 164, B3013 (2017).

2. L. Sang, M. Liao, and M. Sumiya, Sensors, 13, 10482 (2013).

3. A. Tereshchenko, M. Bechelany, R. Viter, V. Khranovskyy, V. Smyntyna, N. Starodub, and R. Yakimova, Sens. Actuators B: Chemical, 229, 664 (2016).

4. M. R. Alenezi, A. S. Alshammari, K. D. G. I. Jayawardena, M. J. Beliatis, S. J. Henley, and S. R. P. Silva, J. Phys. Chem. C, 117, 17850 (2013).

5. A. J. Morfa, B. I. MacDonald, J. Subbiah, and J. J. Jasieniak, Sol. Energ. Mat. Sol. Cells, 124, 211 (2014).

6. E. Espid and F. Taghipour, ECS J. Solid State Sci. Technol., 7, Q3089 (2018).
7. J.-C. Lin, B.-R. Huang, and T.-C. Lin, J. Electrochem. Soc., 160, H509 (2013).

8. R. Martins, R. Igreja, I. Ferreira, A. Marques, A. Pimentel, A. Goncalves, and E. Fortunato, Mater. Sci. Eng. B, 118, 135 (2005).

9. J. Cheng and K. M. Poduska, Nanomater., 3, 317 (2013).

10. J. Cheng, M. A. Rasheed, and K. M. Poduska, ECS J. Solid State Sci. Technol., 2, Q23 (2013).

11. L. B. Rockland, Anal. Chem., 32, 1375 (1960).

12. A. S. Bondarenko and G. A. Ragoisha, In Progress in Chemometrics Research; A. L. Pomerantsev, Ed.; Nova Science Publishers, 2005; Chapter software is available online at http://www.abc.chemistry.bsu.by/vi/analyser/, pp 89.

13. C. Soci, A. Zhang, B. Xiang, S. A. Dayeh, D. P. R. Aplin, J. Park, X. Y. Bao, Y. H. Lo, and D. Wang, Nano Lett., 7, 1003 (2007)

14. J. Cheng and K. M. Poduska, ECS J. Solid State Sci. Technol., 3, P133 (2014).

15. J. L. Lyons, J. B. Varley, D. Steiauf, A. Janotti, and C. G. Van de Walle, J. Appl. Phys., 122, 035704 (2017). 